\title{
Kikuchi Fujimoto as an Initial Presentation of Systemic Lupus Erythromatosis
}

\section{Srijana Basnet, Laxman Shrestha and Prabina Shrestha}

Department of Paediatrics, Institute of Medicine, Maharajgunj, Kathmandu, Nepal

\section{Correspondence:}

Srijana Basnet

Department of Paediatrics,

Institute of Medicine,

Maharajgunj,

Kathmandu, Nepal

Email: drsrijanabasnet@yahoomail.com

DOI: $10.3126 /$ jnps.v41i1.29519

Submitted on: $2020-06-24$

Accepted on: 2021-02-15

Acknowledgements: None

Funding: Nil

Conflict of Interest: None declared

Permission from IRB: Yes
To cite this article: Basnet $\mathrm{S}$, Shrestha $\mathrm{L}$, Shrestha P. Kikuchi Fujimoto as an Initial Presentation of Systemic Lupus Erythromatosis. J Nepal Paediatr Soc. 2021;41(1):99-102.

\section{ABSTRACT}

Kikuchi-Fujimoto Disease (KFD) is a rare benign, condition of necrotising histiocytic lymphadenitis. In this case report, we discuss a case of 10 year old male patient who presented with a fever, rash and generalised lymphadenopathy that was not attributable to the more common causes. Axillary lymph node biopsy confirmed the diagnosis of KFD. Treatment with prednisolone improved his symptoms but after six months he had recurrence of his symptoms. He was investigated again and finally met diagnostic criteria for SLE. This case report highlights importance of close follow up in a child with KFD.

Key words: Fever; Lymphadenopathy; Lymph nodes 


\section{INTRODUCTION}

Kikuchi-Fujimoto disease (KFD) is a rare benign disease with clinical presentation of fever and cervical lymphadenopathy. This condition is confirmed histologically by histiocytic necrotising lymphadenitis without granulocytic infiltration. It was initially described by Japanese pathologists Kikuchi and Fujimoto in 1972. ${ }^{1}$ Its etiology has not yet been fully determined. However, it is believed to be of viral origin and most probable viruses are EBV, HHV6 and 8. An autoimmune etiology is also likely as it has been reported in association with SLE. ${ }^{2,3}$ Females are affected more often than males and the disease is more prevalent in Asia. Only a limited number of cases are found in the literature showing an association between KFD and SLE in male patients. Here, we report a rare case of KFD in a Nepalese boy, who was finally diagnosed as SLE.

\section{CASE REPORT}

A 10 years old male child from Janakpur, Nepal presented to us with one month history of intermittent fever, responding to anti-pyretic. After one week of fever, he developed neck swelling and rashes over trunk and extremities. There was history of profuse sweating during night time. He had lost significant weight over the past month. There was no history of contact with tuberculosis or arthritis, mouth ulcers, drug intake and atopy. $\mathrm{He}$ did not have any dental problem or odynophagia.

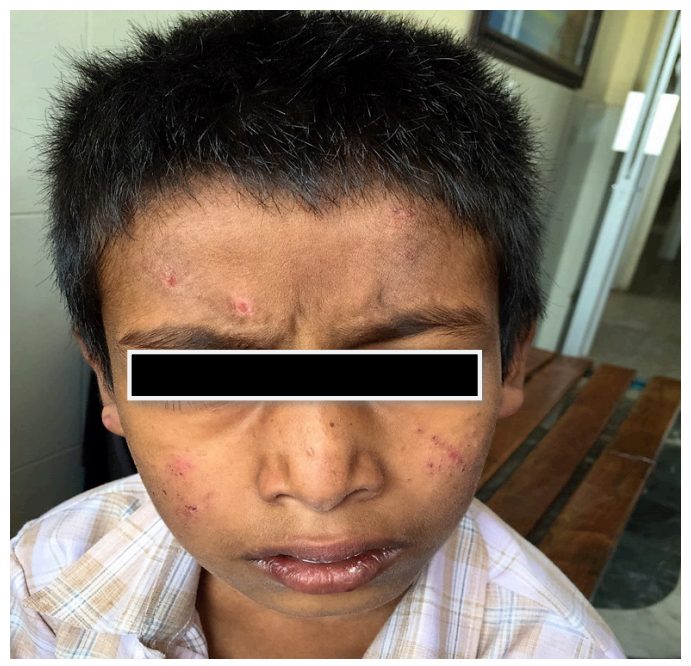

Figure 1. Picture showing rashes over the face
During presentation, he was febrile. There were multiple lymph nodes with largest measuring $2 \mathrm{~cm}$ $\times 2 \mathrm{~cm}$ which were non tender, discrete, mobile and palpable in the neck, axillary and inguinal regions. Multiple erythematous papular rashes noted over face and over trunk which were crusted with central scab formation and turned to perilesional hypopigmented area, over next five days. He had hepatomegaly of $4 \mathrm{~cm}$ below the right costal margin. There was no splenomegaly nor bony tenderness.

His haemoglobin was $12.4 \mathrm{gm} \%$, TLC $5,680 / \mathrm{mm}^{3}$ with a normal differential count, platelet count was 197,000/ $\mathrm{mm}^{3}$ and ESR $46 \mathrm{~mm}$ in the first hour. No abnormal cells were seen on peripheral smear. Widal test was negative. Chest $\mathrm{X}$-ray and abdominal ultrasound were normal. ANA and HIV serology were negative. Mantoux test was negative. His LDH was $1482 \mathrm{U} / \mathrm{L}$, ALP $201 \mathrm{U} / \mathrm{L}$, uric acid was $164 \mu \mathrm{mol} / \mathrm{L}$, calcium $1.8 \mu \mathrm{mol} / \mathrm{L}$, phosphorous $3.1 \mathrm{mg} / \mathrm{dL}, \mathrm{SGOT} / \mathrm{SGPT}$ were normal. Lymph node FNAC was suggestive of reactive lymphadenitis. Lymph node biopsy was also done from axillary lymph node. The excised lymph nodes showed partial effacement of architecture by necrosis. There was alternating dark and pale areas. Pale zone constituted either necrosis or macrophages. There were crescentic macrophages containing karyorrhectic debris. Dark zone constituted small lymphocytes and large lymphoid cells. No granulomas or malignancy were seen. These

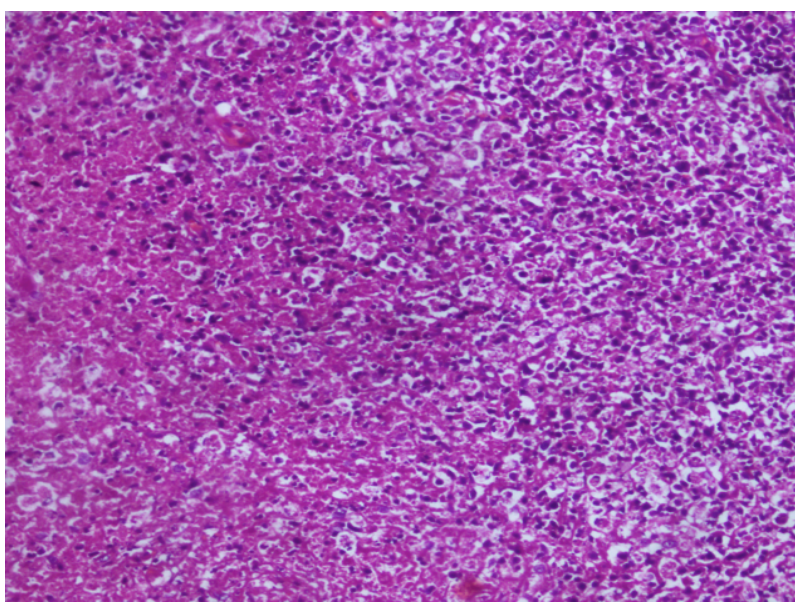

Figure 2. Lymph node histopathology with arrows showing necrosis with multiple cells crescentic macrophages (histiocytes) with karyorrhectic nuclei, predominant lymphocytosis with typical absence of neutrophilsconsistent with Kikuchi Fujimoto Disease 
findings were consistent with necrotising histiocytic lymphadenitis (Kikuchi lymphadenitis).

He was given prednisolone $1 \mathrm{mg} / \mathrm{kg} / \mathrm{d}$ for two weeks. In next two weeks, he became afebrile. The lymph nodes size decreased gradually. The patient had been counselled for regular follow-up. Steroid was gradually tapered and stopped over next two weeks.

After six months, he again developed fever and rashes. His ANA and dsDNA were sent which were positive. Hence, he was diagnosed as SLE and started on prednisolone. His complete blood count, renal function and urine examination were normal. The boy was also started on hydroxychloroquine and advised for use of sunscreen.

\section{DISCUSSION}

Initial clinical presentation of this case resembles that of infectious origin like viral or rickettsial infection. On clinical suspicion, a course of doxycycline was given for one week but there was no clinical response. The clinical presentation even raised suspicion of lymphoma or tuberculosis but investigations refute these diagnoses. Systemic lupus erythematosus was also considered strongly but the clinical and laboratory evaluation did not fulfil ACR or SLICC criteria for the diagnosis. ${ }^{4}$ In the mean time the availability of biopsy report confirmed the diagnosis of unusual KFD.

The younger age of presentation, generalised lymphadenopathy and presence of rashes observed in this case, are unusual presentation in KFD. KFD commonly affects young female adults. There are only few case reports of this disease in children. Less than one third of the patients present with rash, weight loss and hepatomegaly. In Taiwan, Chuang et al. studied 64 patients younger than 18 years with tissue diagnosis of KFD. ${ }^{5}$ They also observed generalised lymphadenopathy in only one patient. In a retrospective study from South India, 53 children were reported with KFD, six $(11.3 \%)$ of whom had rashes. ${ }^{6}$
There is no specific treatment for KFD. In cases in which KFD co-occurs with SLE or in complicated cases with increased lactate dehydrogenase, and serum antinuclear antibody titers, and presence of neurological signs, use of corticosteroids or immunosuppressant has been recommended to prevent a fatal outcome. ${ }^{7,8}$ Since our patient had high $\mathrm{LDH}$, he was treated with steroid initially for two weeks and he remained well for next six months.

Histopathological findings of affected lymph nodes reveal characteristic findings of KFD. There are three main patterns identified with KFD: proliferative; necrotising and xanthomatous. The proliferative picture is seen in approximately a third of cases and has a dominant inflammatory infiltrate. Cellular protein structures have been noted in the cytoplasm of lymphocytes and histiocytes have also been found in those cells of patients with SLE. This adds strength to the hypothesis that KFD is a self-limiting SLE-like disorder. According to Kucukardali et al., SLEassociated KFD is more common in Asian patients than in European patients. Twenty-eight patients were studied; 18 had simultaneous SLE and KFD, six presented with SLE after KFD diagnosis, and four were previously diagnosed with SLE. ${ }^{9}$ The association between these two disorders are not completely understood. The clinical features are similar, and differentiation between them is based on lymph node histopathology. ${ }^{10}$ The absence of hematoxylin bodies and neutrophils indicates KFD rather than SLE, and our patient showed definite features of KFD.

\section{CONCLUSIONS}

KFD is an unusual cause of cervical lymphadenopathy. Presence of rash and generalised lymphadenopathy can occur infrequently in KFD. Lymph node biopsy confirms the diagnosis. Patients with KFD needs evaluation for SLE during first presentation and follow up.

\section{REFERENCES}

1. Perry A, Mand Choi SM. Kikuchi-Fujimoto Disease: A Review. Arch Pathol Lab Med. 2018 Nov;142(11): $1341-6$. DOI10.5858/arpa.2018-0219-RA. 
2. Diego FB, Diehl FA, Salinas MJH, Riva V, Diller A, Lemos PA. Kikuchi - Fujimoto disease and systemic lupus erythematosus. Int Med Case Rep J. 2016;29:9163-7. DOI: 10.2147/IMCRJ.S106396.

3. Dumas G, Prendki ÃV, Haroche J, Amoura Z, Cacoub P, Galicier L et al. Kikuchi-Fujimoto Disease Retrospective Study of 91 Cases and Review of the Literature. Medicine (Baltimore). 2014 Nov;93(24):372-82. DOI:10.1097/ MD.0000000000000220.

4. Petri M, Orbai AM, Alarcon GS, Gordon C, Merrill JT, Fortin PR et al. Derivation and validation of the Systemic Lupus International Collaborating Clinics classification criteria for systemic lupus erythematosus. Arthritis Rheum. 2012 Aug;64(8):2677-86. DOI: 10.1002/art.34473.

5. Chuang CH, Yan DC, Chiu CH, Huang YC, Lin PY, Chen CY et al. Clinical and laboratory manifestations of Kikuchi's disease in children and differences between patients with and without prolonged fever. Pediatr Infect Dis J. 2005 Jun; 24(6):551-4. DOI: 10.1097/01.inf.0000167246.24500.21

6. Punnen A, Kanagalakshmi, Manipadam MT, VergheseVP, Mathew LG, Kumar S. Clinicopathologic study of Kikuchi's disease in children in a tertiary hospital in South India. Int J Contemp Pediatr. 2019;6:664-9. DOI: http:// dx.doi.org/10.18203/2349-3291.ijcp20190708

7. Chan JK, Wong KC, Ng CS. A fatal case of multicentric Kikuchi's histiocytic necrotizing lymphadenitis. Cancer. 1989 May 1;63(9):1856-62. PMID: 2784712

8. Lin SH, Ko WS, Lee HS, Hwang WS. Kikuchi's disease associated with lupus-like syndrome - a fatal case. J Rheumatol. 1992;19:1995-6. PMID: 1294758

9. Kucukardali Y, Solmazgul E, Kunter E, Oncul O, Yildirim S, Kaplan M. Kikuchi-Fujimoto disease: analysis of 244 cases. Clin Rheumatol. 2007 Jan;26(1):50-4. DOI: 10.1007/s10067-006-0230-5.

10. Chamulak GA, Brynes RK and Nathwani BN. Kikuchi- Fujimoto disease mimicking malignant lymphoma. Am J Surg Pathol.1990;14(6):514-23. DOI: 10.1097/00000478-199006000-00002. 